\title{
PHYSICAL CAUSES OF ENERGY-DENSITY INHOMOGENIZATION AND STABILITY OF ENERGY-DENSITY HOMOGENEITY IN RELATIVISTIC SELF-GRAVITATING FLUIDS
}

\author{
L. Herrera隹 \\ Departamento de Física Teórica e Historia de la Ciencia, Universidad del País Vasco, Bilbao, Spain
}

(Dated: September 24, 2018)

\begin{abstract}
We identify the factors responsible for the appearance of energy-density inhomogeneities in a self-gravitating fluid, and describe the evolution of those factors from an initially homogeneous distribution. It is shown that a specific combination of the Weyl tensor and/or local anisotropy of pressure and/or dissipative fluxes entails the formation of energy-density inhomogeneities. Different cases are analyzed in detail and in the particular case of dissipative fluids, the role of relaxational processes as well as non-local effects are brought out.
\end{abstract}

PACS numbers: 04.40.Cv, 04.40.Dg, 04.40.Nr

Keywords: Relativistic fluids, general relativity, dissipative systems.

\section{INTRODUCTION}

The role of energy-density inhomogeneity in the collapse of self-gravitating fluids is quite relevant and has been extensively discussed in the literature (see [1] $-[8]$ and references therein).

Furthermore the Penrose's proposal [9] to define a gravitational arrow of time in terms of the Weyl tensor, is based on the particularly simple relation between the Weyl tensor and density inhomogeneity, for perfect fluids. However the fact that such a relationship is no longer valid in the presence of local anisotropy of the pressure and/or dissipative processes, and/or electric charge distribution [10 12], explains its failure in scenarios where the above-mentioned factors are present [13 15]. Therefore, since the rationale behind Penrose's idea is that tidal forces tend to make the gravitating fluid more inhomogeneous as the evolution proceeds, thereby indicating the sense of time, it should be clear that all factors asociated to energy-density inhomogenity (and not only the Weyl tensor) should be present in any definition of the gravitational arrow of time a la Penrose.

However, in spite of its relevance, it is unclear yet how different physical phenomena affect and, more precisely, produce energy- density inhomogeneities. The pertinence of such a question is supported by the arguments above.

It is the purpose of this work to answer to the above mentioned question. It entails in fact three different (but related) questions, namely:

- What aspects of the fluid distribution are related (and how) to the existence of energy-density inhomogeneities ?

- How those factors evolve, starting with an initially homogeneous distribution?

\footnotetext{
*Also at U.C.V., Caracas

${ }^{\dagger}$ Electronic address: laherrera@cantv.net.ve
}

this last question in turn leads to a third relevant question:

- Under which conditions an initially homogeneous configuration remains homogeneous all along its evolution (stability problem)?

Our procedure will be heavily rely on two differential equations relating the Weyl tensor to different physical variables. On of them is an evolution equation containing time derivatives of those variables, whereas the other is a constraint equation implying spatial derivatives. As far as we know these equations were first derived by Ellis [16, 17] for configurations without any specific symmetry, afterwards they have been reobtained and used by different authors (see for example [10], [18]-[22]).

We shall consider general fluid distributions endowed with anisotropic pressure and dissipating energy during its evolution. The specific physical (microscopic) phenomena behind these fluid characteristics will be not discussed here, instead we shall be concerned only by the macroscopic (hydrodynamic) manifestations of those phenomena (arguments to justify such kind of fluid distributions may be found in [23]-[25] ).

Dissipation processes are usually treated invoking two possible (opposite) approximations: diffusion and streaming out, so we shall do here. In the case of diffusion approximation a causal transport equation will be used, allowing to bring out the effects of pre-relaxational phenomena.

In the specific case of localized configurations we have to assume that our fluid distribution is bounded by a spherical surface. In order to avoid thin shells on such a boundary surface Darmois [26] conditions should be imposed. 


\section{ENERGY-MOMENTUM TENSOR, RELEVANT VARIABLES AND FIELD EQUATIONS}

As is usually assumed in the study of self-gravitating compact objects, we shall consider that deviations from spherical symmetry are incidental rather than basic features of the process involved. Accordingly we shall restrain ourselves to spherically symmetric fluid distributions.

Thus, we consider a spherically symmetric distribution of collapsing fluid, bounded by a spherical surface $\Sigma$. The fluid is assumed to be locally anisotropic (principal stresses unequal) and undergoing dissipation in the form of heat flow (to model dissipation in the diffusion approximation) and null radiation (to model dissipation in the free streaming approximation).

Choosing comoving coordinates inside $\Sigma$, the general interior metric can be written

$$
d s^{2}=-A^{2} d t^{2}+B^{2} d r^{2}+R^{2}\left(d \theta^{2}+\sin ^{2} \theta d \phi^{2}\right),
$$

where $A, B$ and $R$ are functions of $t$ and $r$ and are assumed positive. We number the coordinates $x^{0}=t$, $x^{1}=r, x^{2}=\theta$ and $x^{3}=\phi$.

The matter energy-momentum $T_{\alpha \beta}$ inside $\Sigma$ has the form

$$
\begin{aligned}
T_{\alpha \beta} & =\left(\mu+P_{\perp}\right) V_{\alpha} V_{\beta}+P_{\perp} g_{\alpha \beta}+\left(P_{r}-P_{\perp}\right) \chi_{\alpha} \chi_{\beta}+q_{\alpha} V_{\beta} \\
& +V_{\alpha} q_{\beta}+\epsilon l_{\alpha} l_{\beta}
\end{aligned}
$$

where $\mu$ is the energy density, $P_{r}$ the radial pressure, $P_{\perp}$ the tangential pressure, $q^{\alpha}$ the heat flux describing dissipation in the diffusion approximation, $\epsilon$ the energy density of the null fluid describing dissipation in the free streaming approximation, $V^{\alpha}$ the four velocity of the fluid, $\chi^{\alpha}$ a unit four vector along the radial direction and $l^{\alpha}$ a radial null four vector. These quantities satisfy

$$
V^{\alpha} V_{\alpha}=-1, \quad V^{\alpha} q_{\alpha}=0, \quad \chi^{\alpha} \chi_{\alpha}=1,
$$

and

$$
\chi^{\alpha} V_{\alpha}=0, \quad l^{\alpha} V_{\alpha}=-1, \quad l^{\alpha} l_{\alpha}=0
$$

The four-acceleration $a_{\alpha}$ and the expansion $\Theta$ of the fluid are given by

$$
a_{\alpha}=V_{\alpha ; \beta} V^{\beta}, \quad \Theta=V_{; \alpha}^{\alpha} .
$$

and its shear $\sigma_{\alpha \beta}$ by

$$
\sigma_{\alpha \beta}=V_{(\alpha ; \beta)}+a_{(\alpha} V_{\beta)}-\frac{1}{3} \Theta h_{\alpha \beta}
$$

where $h_{\alpha \beta}=g_{\alpha \beta}+V_{\alpha} V_{\beta}$.

We do not explicitly add bulk viscosity and/or shear viscosity to the system because they can be absorbed into the radial and tangential pressures, $P_{r}$ and $P_{\perp}$, of the collapsing fluid.

Since we assumed the metric (1) comoving then

$$
\begin{gathered}
V^{\alpha}=A^{-1} \delta_{0}^{\alpha}, \quad q^{\alpha}=q B^{-1} \delta_{1}^{\alpha}, \\
l^{\alpha}=A^{-1} \delta_{0}^{\alpha}+B^{-1} \delta_{1}^{\alpha}, \quad \chi^{\alpha}=B^{-1} \delta_{1}^{\alpha},
\end{gathered}
$$

where $q$ is a function of $t$ and $r$ satisfying $q^{\alpha}=q \chi^{\alpha}$.

From (5) with (7) we have for the acceleration and its scalar $a$,

$$
a_{1}=\frac{A^{\prime}}{A}, \quad a^{2}=a^{\alpha} a_{\alpha}=\left(\frac{A^{\prime}}{A B}\right)^{2},
$$

where $a^{\alpha}=a \chi^{\alpha}$, and for the expansion

$$
\Theta=\frac{1}{A}\left(\frac{\dot{B}}{B}+2 \frac{\dot{R}}{R}\right)
$$

where the prime stands for $r$ differentiation and the dot stands for differentiation with respect to $t$. With (7) we obtain for the shear (6) its non zero components

$$
\sigma_{11}=\frac{2}{3} B^{2} \sigma, \quad \sigma_{22}=\frac{\sigma_{33}}{\sin ^{2} \theta}=-\frac{1}{3} R^{2} \sigma,
$$

and its scalar

$$
\sigma^{\alpha \beta} \sigma_{\alpha \beta}=\frac{2}{3} \sigma^{2}
$$

where

$$
\sigma=\frac{1}{A}\left(\frac{\dot{B}}{B}-\frac{\dot{R}}{R}\right)
$$

Then, the shear tensor can be written as

$$
\sigma_{\alpha \beta}=\sigma\left(\chi_{\alpha} \chi_{\beta}-\frac{1}{3} h_{\alpha \beta}\right) .
$$

\section{A. The Einstein equations} by

Einstein's field equations for the metric (11) are given

$$
G_{\alpha \beta}=8 \pi T_{\alpha \beta}
$$

its non zero components with (11), (2) and (7) become

$$
8 \pi T_{00}=8 \pi(\mu+\epsilon) A^{2}=\left(2 \frac{\dot{B}}{B}+\frac{\dot{R}}{R}\right) \frac{\dot{R}}{R}-\left(\frac{A}{B}\right)^{2}\left[2 \frac{R^{\prime \prime}}{R}+\left(\frac{R^{\prime}}{R}\right)^{2}-2 \frac{B^{\prime}}{B} \frac{R^{\prime}}{R}-\left(\frac{B}{R}\right)^{2}\right]
$$




$$
8 \pi T_{01}=-8 \pi(q+\epsilon) A B=-2\left(\frac{\dot{R}^{\prime}}{R}-\frac{\dot{B}}{B} \frac{R^{\prime}}{R}-\frac{\dot{R}}{R} \frac{A^{\prime}}{A}\right)
$$

$$
8 \pi T_{11}=8 \pi\left(P_{r}+\epsilon\right) B^{2}=-\left(\frac{B}{A}\right)^{2}\left[2 \frac{\ddot{R}}{R}-\left(2 \frac{\dot{A}}{A}-\frac{\dot{R}}{R}\right) \frac{\dot{R}}{R}\right]+\left(2 \frac{A^{\prime}}{A}+\frac{R^{\prime}}{R}\right) \frac{R^{\prime}}{R}-\left(\frac{B}{R}\right)^{2}
$$

$$
\begin{aligned}
8 \pi T_{22}=\frac{8 \pi}{\sin ^{2} \theta} T_{33}=8 \pi P_{\perp} & R^{2}=-\left(\frac{R}{A}\right)^{2}\left[\frac{\ddot{B}}{B}+\frac{\ddot{R}}{R}-\frac{\dot{A}}{A}\left(\frac{\dot{B}}{B}+\frac{\dot{R}}{R}\right)+\frac{\dot{B}}{B} \frac{\dot{R}}{R}\right] \\
+ & \left(\frac{R}{B}\right)^{2}\left[\frac{A^{\prime \prime}}{A}+\frac{R^{\prime \prime}}{R}-\frac{A^{\prime}}{A} \frac{B^{\prime}}{B}+\left(\frac{A^{\prime}}{A}-\frac{B^{\prime}}{B}\right) \frac{R^{\prime}}{R}\right] .
\end{aligned}
$$

\section{B. The mass function}

Let us now introduce the mass function $m(t, r)$ [27] (see also [28]), defined by

$$
m=\frac{R^{3}}{2} R_{23}^{23}=\frac{R}{2}\left[\left(\frac{\dot{R}}{A}\right)^{2}-\left(\frac{R^{\prime}}{B}\right)^{2}+1\right] .
$$

Following Misner and Sharp [27], it is useful to define the proper time derivative $D_{T}$ given by

$$
D_{T}=\frac{1}{A} \frac{\partial}{\partial t}
$$

and the " $R$ " derivative $D_{R}$,

$$
D_{R}=\frac{1}{R^{\prime}} \frac{\partial}{\partial r},
$$

where $R$ defines the areal radius of a spherical surface inside $\Sigma$ ( as measured from its area).

Using (20) we can define the velocity $U$ of the collapsing fluid as the variation of the areal radius with respect to proper time, i.e.

$$
U=D_{T} R
$$

Then (19) can be rewritten as

$$
E \equiv \frac{R^{\prime}}{B}=\left(1+U^{2}-\frac{2 m}{R}\right)^{1 / 2}
$$

Using (15)-(17) with (20) and (21) we obtain from (19)

$$
D_{T} m=-4 \pi\left[\left(\tilde{P}_{r}-\frac{4}{3} \eta \sigma\right) U+\tilde{q} E\right] R^{2},
$$

and

$$
D_{R} m=4 \pi\left(\tilde{\mu}+\tilde{q} \frac{U}{E}\right) R^{2},
$$

which implies

$$
m=4 \pi \int_{0}^{r}\left(\tilde{\mu}+\tilde{q} \frac{U}{E}\right) R^{2} R^{\prime} d r
$$

(assuming a regular centre to the distribution, so $m(0)=$ $0)$. Integrating (26) we find

$$
\frac{3 m}{R^{3}}=4 \pi \tilde{\mu}-\frac{4 \pi}{R^{3}} \int_{0}^{r} R^{3}\left(D_{R} \tilde{\mu}-3 \tilde{q} \frac{U}{R E}\right) R^{\prime} d r .
$$

with

$$
\tilde{\mu}=\mu+\epsilon,
$$

$$
\tilde{P}_{r}=P_{r}+\epsilon,
$$

$$
\tilde{q}=q+\epsilon,
$$




\section{Weyl tensor}

The Weyl tensor is defined through the Riemann tensor $R_{\alpha \beta \mu}^{\rho}$, the Ricci tensor $R_{\alpha \beta}$ and the curvature scalar $\mathcal{R}$, as:

$$
\begin{aligned}
C_{\alpha \beta \mu}^{\rho}= & R_{\alpha \beta \mu}^{\rho}-\frac{1}{2} R_{\beta}^{\rho} g_{\alpha \mu}+\frac{1}{2} R_{\alpha \beta} \delta_{\mu}^{\rho}-\frac{1}{2} R_{\alpha \mu} \delta_{\beta}^{\rho} \\
& +\frac{1}{2} R_{\mu}^{\rho} g_{\alpha \beta}+\frac{1}{6} \mathcal{R}\left(\delta_{\beta}^{\rho} g_{\alpha \mu}-g_{\alpha \beta} \delta_{\mu}^{\rho}\right) .
\end{aligned}
$$

The electric part of Weyl tensor is defined by

$$
E_{\alpha \beta}=C_{\alpha \mu \beta \nu} V^{\mu} V^{\nu}
$$

with the following non-vanishing components

$$
\begin{aligned}
& E_{11}=\frac{2}{3} B^{2} \mathcal{E}, \\
& E_{22}=-\frac{1}{3} R^{2} \mathcal{E}, \\
& E_{33}=E_{22} \sin ^{2} \theta,
\end{aligned}
$$

where

$$
\begin{gathered}
\mathcal{E}=\frac{1}{2}\left[\frac{\ddot{R}}{R}-\frac{\ddot{B}}{B}-\left(\frac{\dot{R}}{R}-\frac{\dot{B}}{B}\right) \frac{\dot{R}}{R}\right] \\
+\frac{1}{2 B^{2}}\left[-\frac{R^{\prime \prime}}{R}+\left(\frac{B^{\prime}}{B}+\frac{R^{\prime}}{R}\right) \frac{R^{\prime}}{R}\right]-\frac{1}{2 R^{2}} .
\end{gathered}
$$

Observe that we may also write $E_{\alpha \beta}$ as:

$$
E_{\alpha \beta}=\mathcal{E}\left(\chi_{\alpha} \chi_{\beta}-\frac{1}{3} h_{\alpha \beta}\right)
$$

Finally, using (15), (17), (18) with (19) and (31) we obtain

$$
\frac{3 m}{R^{3}}=4 \pi(\tilde{\mu}-\Pi)-\mathcal{E},
$$

with

$$
\Pi=\tilde{P}_{r}-P_{\perp}
$$

\section{BIANCHI IDENTITIES AND ELLIS EQUATIONS}

As mentioned in the Introduction two differential equations for the Weyl tensor will play a central role in our work, these two equations originally found by Ellis [16, 17] are here reobtained following the procedure adopted in [10]. Beside these two equations we shall also need the Bianchi identities, which for the system under consideration have two independent components which read (see [29] for details):

$$
\dot{\tilde{\mu}}+\left(\tilde{\mu}+\tilde{P}_{r}\right) \frac{\dot{B}}{B}+2\left(\tilde{\mu}+P_{\perp}\right) \frac{\dot{R}}{R}+\frac{\tilde{q}^{\prime} A}{B}+2 \tilde{q} \frac{(A R)^{\prime}}{B R}=0,
$$

and

$$
\dot{\tilde{q}}+\left(\tilde{P}_{r}\right)^{\prime} \frac{A}{B}+2 \tilde{q}\left(\frac{\dot{B}}{B}+\frac{\dot{R}}{R}\right)+\left(\tilde{\mu}+\tilde{P}_{r}\right) \frac{A^{\prime}}{B}+2 \Pi \frac{A R^{\prime}}{B R}=0
$$

Finally, the following equations for the Weyl tensor may be derived (e.g. see [10] for details, but notice changes in notation)

$$
[\mathcal{E}-4 \pi(\tilde{\mu}-\Pi)]=\frac{3 \dot{R}}{R}\left(\frac{3 m}{R^{3}}+4 \pi \tilde{P}_{r}\right)+12 \pi \tilde{q} \frac{A R^{\prime}}{B R}=\frac{3 \dot{R}}{R}\left[4 \pi\left(\tilde{\mu}+P_{\perp}\right)-\mathcal{E}\right]+12 \pi \tilde{q} \frac{A R^{\prime}}{B R}
$$




$$
[\mathcal{E}-4 \pi(\tilde{\mu}-\Pi)]^{\prime}=-\frac{3 R^{\prime}}{R}(\mathcal{E}+4 \pi \Pi)-12 \pi \tilde{q} \frac{B \dot{R}}{A R}=-\frac{3 R^{\prime}}{R}\left[4 \pi \tilde{\mu}-\frac{3 m}{R^{3}}\right]-12 \pi \tilde{q} \frac{B \dot{R}}{A R}
$$

\section{THE TRANSPORT EQUATION}

In the diffusion approximation $(\epsilon=0, \tilde{q}=q)$, we shall need a transport equation derived from a causal dissipative theory ( e.g. the Müller-Israel-Stewart second order phenomenological theory for dissipative fluids [30-33]).

Indeed, as it is already well known the Maxwell-Fourier law for radiation flux leads to a parabolic equation (diffusion equation) which predicts propagation of perturbations with infinite speed (see [34]-37] and references therein). This simple fact is at the origin of the pathologies 38 found in the approaches of Eckart 39] and Landau [40] for relativistic dissipative processes. To overcome such difficulties, various relativistic theories with non-vanishing relaxation times have been proposed in the past $30-33,41,42$. Although the final word on this issue has not yet been said, the important point is that all these theories provide a heat transport equation which is not of Maxwell-Fourier type but of Cattaneo type [43], leading thereby to a hyperbolic equation for the propagation of thermal perturbations.

A key quantity in these theories is the relaxation time $\tau$ of the corresponding dissipative process. This positivedefinite quantity has a distinct physical meaning, namely the time taken by the system to return spontaneously to the steady state (whether of thermodynamic equilibrium or not) after it has been suddenly removed from it. Therefore, when studying transient regimes, i.e., the evolution from a steady-state situation to a new one, $\tau$ cannot be neglected. In fact, leaving aside that parabolic theories are necessarily non-causal, it is obvious that whenever the time scale of the problem under consideration becomes of the order of (or smaller) than the relaxation time, the latter cannot be ignored. Indeed, neglecting the relaxation time ammounts -in this situationto disregarding the whole problem under consideration.

It is worth mentioning that sometimes in the past it has been argued that dissipative processes with relaxation times comparable to the characteristic time of the system are out of the hydrodynamic regime. However, the concept of hydrodynamic regime involves the ratio between the mean free path of fluid particles and the characteristic length of the system. When this ratio is lower that unity, the fluid is within the hydrodynamic regime. When it is larger than unity, the regime becomes Knudsen's. In the latter case the fluid is no longer a continuum and even hyperbolic theories cease to be realiable.

Therefore that argument can be valid only if the particles making up the fluid are the same ones that transport the heat. However, this is never the case. Specifically, for a neutron star, $\tau$ is of the order of the scattering time between electrons (which carry the heat) but this fact is not an obstacle (no matter how large the mean free path of these electrons may be) to consider the neutron star as formed by a Fermi fluid of degenerate neutrons. The same is true for the second sound in superfluid Helium and solids, and for almost any ordinary fluid. In brief, the hydrodynamic regime refers to fluid particles that not necessarily (and as a matter of fact, almost never) transport the heat. Therefore large relaxation times (large mean free paths of particles involved in heat transport) does not imply a departure from the hydrodynamic regime (this fact has been streseed before [44], but it is usually overlooked).

The corresponding transport equation for the heat flux reads

$\tau h^{\alpha \beta} V^{\gamma} q_{\beta ; \gamma}+q^{\alpha}=-\kappa h^{\alpha \beta}\left(T_{, \beta}+T a_{\beta}\right)-\frac{1}{2} \kappa T^{2}\left(\frac{\tau V^{\beta}}{\kappa T^{2}}\right)_{; \beta} q^{\alpha}$,

where $\kappa$ denotes the thermal conductivity, and $T$ and $\tau$ denote temperature and relaxation time respectively. Observe that, due to the symmetry of the problem, equation (38) only has one independent component, which may be written as

$$
\tau \dot{q}=-\frac{1}{2} \kappa q T^{2}\left(\frac{\tau}{\kappa T^{2}}\right)^{\cdot}-\frac{1}{2} \tau q \Theta A-\frac{\kappa}{B}(T A)^{\prime}-q A .
$$

In the case $\tau=0$ we recover the Eckart-Landau equation.

In the truncated version of the theory, the last term in (38) is absent (see for example [45]), and (39) becomes

$$
\tau \dot{q}+q A=-\frac{\kappa}{B}(T A)^{\prime} .
$$

\section{CAUSES OF INHOMOGENIZATION AND STABILITY OF HOMOGENEOUS ENERGY-DENSITY CONDITION}

We shall now use the two equations for the Weyl tensor introduced in section III in order to answer to the main questions raised in this work. We shall proceed by considering particular cases, in an increasing order of complexity

\section{A. Non-dissipative dust}

In this case $P_{r}=P_{\perp}=\tilde{q}=0$ and from the fact that the fluid is geodesic as follows from (35) we can put $A=1$. 
Then equations (36) and (37) become

$$
(\mathcal{E}-4 \pi \mu)+\frac{3 \dot{R}}{R}(\mathcal{E}-4 \pi \mu)=0,
$$

and

$$
(\mathcal{E}-4 \pi \mu)^{\prime}=-\frac{3 R^{\prime}}{R} \mathcal{E} .
$$

From this last equation it follows at once that $\mathcal{E}=0 \leftrightarrow$ $\mu^{\prime}=0$ a well known result implying that energy-density inhomogeneities in this particular case are controlled by the Weyl tensor alone.

Next, using (12) and (34) in (41), this last equation takes the form

$$
\dot{\mathcal{E}}+\frac{3 \mathcal{E} \dot{R}}{R}=-4 \pi \mu \sigma,
$$

whose solution may be written as

$$
\mathcal{E}=-\frac{4 \pi \int_{0}^{t} \mu \sigma R^{3} d t}{R^{3}}
$$

where we have chosen the integration function such that $\mathcal{E}(0, r)=0$.

It is worth noticing that in this particular case (nondissipative dust), conformal flatness and shear-free conditions imply each other as it follows from (43) and the evolution equation for the shear, which reads (see [22, 46])

$$
\dot{\sigma}+\frac{\sigma^{2}}{3}+\frac{2 \Theta \sigma}{3}=-\mathcal{E} .
$$

Thus, an initially homogeneous distribution of nondissipative dust will remain so iff it remains conformally flat, which in turn implies that it remains shear-free. In other words the problem of the stability of the energydensity homogenity reduces to the stability of conformal flatness or to the stability of the shear-free condition.

\section{B. Non-dissipative locally isotropic fluid}

The next case in increasing order of complexity, corresponds to a non-dissipative isotropic (in the pressure) fluid. Thus we have $\Pi=\tilde{q}=0, P_{r}=P_{\perp}=P$. Then equations (36) and (37) become

$$
(\mathcal{E}-4 \pi \mu)+\frac{3 \dot{R}}{R}[\mathcal{E}-4 \pi(\mu+P)]=0,
$$

and

$$
(\mathcal{E}-4 \pi \mu)^{\prime}=-\frac{3 R^{\prime}}{R} \mathcal{E} .
$$

As in the previous case we have from (47) that $\mathcal{E}=0 \leftrightarrow$ $\mu^{\prime}=0$, meaning that energy-density inhomogeneities are controlled by the Weyl tensor alone, in this case too. In other words, the problem of the stability of the energydensity homogeneity reduces to that of the stability of the conformal flatness. It is also worth mentioning that in this case the vanishing of Weyl implies shear-free, however the inverse is no true, since the evolution equation for the shear now reads

$$
\mathcal{E}=\frac{a^{\prime}}{B}-\frac{\dot{\sigma}}{A}+a^{2}-\frac{\sigma^{2}}{3}-\frac{2}{3} \Theta \sigma-a \frac{R^{\prime}}{B R} .
$$

Next, using using (12) and (34) in (46), this last equation becomes

$$
\dot{\mathcal{E}}+\frac{3 \mathcal{E} \dot{R}}{R}=-4 \pi(\mu+P) A \sigma .
$$

In the general shearing case we have from (49)

$$
\mathcal{E}=-\frac{4 \pi \int_{0}^{t}(\mu+P) A \sigma R^{3} d t}{R^{3}},
$$

where again we have chosen the integration function such that $\mathcal{E}(0, r)=0$. Obviously, in the case of dust (50) becomes (44).

Thus the stability of the energy-density homogeneity condition is as in the previos case controlled by the shear of the fluid.

However, as mentioned before, even though all spherically symmetric conformally flat spacetimes (without dissipation) and isotropic fluids are shear-free the inverse is not true, and we may assume the fluid to be shear-free without that implying conformal flatness. In this latter case we have from (49)

$$
\mathcal{E}=\frac{f(r)}{R^{3}},
$$

where $f(r)$ is an arbitrary function of $r$ satisfying $f(0)=$ 0 .

Then, if we start with an initially homogeneous configuration at $t=0$, implying $\mathcal{E}(0, r)=0$ then $f(r)=0$ producing $\mathcal{E}=0$ for any $t$ and accordingly the energydensity homogeneous condition will hold all along the evolution.

If instead we assume a small though non-vanishing initial Weyl tensor then it will remain small if the fluid expands. However in the contraction case it may become relevant for sufficiently small $R$. Thus, in the case of contraction the energy-density homogeneity might not be stable in the Lyapounov sense for a shear-free perfect fluid.

\section{Locally anisotropic non-dissipative fluid}

We shall now bring out the role of the pressure anisotropy in the problem under consideration. For that purpose we shall assume $\tilde{q}=0$ but $\Pi \neq 0$. Then equations (36) and (37) become

$$
(\mathcal{E}-4 \pi \mu+4 \pi \Pi)+\frac{3 \dot{R}}{R}\left[\mathcal{E}-4 \pi\left(\mu+P_{\perp}\right)\right]=0,
$$


and

$$
(\mathcal{E}-4 \pi \mu+4 \pi \Pi)^{\prime}+\frac{3 R^{\prime}}{R}(\mathcal{E}+4 \pi \Pi)=0 .
$$

The first remark emerging from (53) is that unlike the previous cases, now the responsible for the existence of energy-density inhomogeneity is not the Weyl tensor alone, but the quantity $\mathcal{E}+4 \pi \Pi$. Indeed, assuming that the fluid distribution is regular at the centre, it follows at once from (53) that $\mathcal{E}+4 \pi \Pi=0 \leftrightarrow \mu^{\prime}=0$.

Next, using (12) and (34) in (52), this last equation becomes

$$
(\mathcal{E}+4 \pi \Pi)^{\dot{ }}+\frac{(3 \mathcal{E}+4 \pi \Pi) \dot{R}}{R}=-4 \pi\left(\mu+P_{r}\right) A \sigma,
$$

thus we have an evolution equation for the quantity responsible for the energy-density inhomogenity. It is worth observing that the quantity $-(\mathcal{E}+4 \pi \Pi)$ is referred to in previous works 21], [46] (notice a difference in notation with respect to the first of these references) as one of the structure scalars $\left(X_{T F}\right)$. It is defined as follows:

Let us introduce the tensor $X_{\alpha \beta}$ defined by:

$$
X_{\alpha \beta}={ }^{*} R_{\alpha \gamma \beta \delta}^{*} V^{\gamma} V^{\delta}=\frac{1}{2} \eta_{\alpha \gamma}{ }^{\epsilon \rho} R_{\epsilon \rho \beta \delta}^{*} V^{\gamma} V^{\delta}
$$

where $R_{\alpha \beta \gamma \delta}^{*}=\frac{1}{2} \eta_{\epsilon \rho \gamma \delta} R_{\alpha \beta}^{\epsilon \rho}$ and $\eta_{\epsilon \rho \gamma \delta}$ denotes the LeviCivita tensor.

Tensor $X_{\alpha \beta}$ may also be expressed through its trace and its trace-free part, as

$$
X_{\alpha \beta}=\frac{1}{3} X_{T} h_{\alpha \beta}+X_{T F}\left(\chi_{\alpha} \chi_{\beta}-\frac{1}{3} h_{\alpha \beta}\right) .
$$

Then from field equations, (56) and using (28) (31) and (32), we obtain (assuming vanishing dissipation)

$$
X_{T F}=-\mathcal{E}-4 \pi \Pi
$$

Therefore the evolution equation (54) in terms of $X_{T F}$ reads

$$
\dot{X}_{T F}+\frac{3 X_{T F} \dot{R}}{R}=-8 \pi \Pi \frac{\dot{R}}{R}+4 \pi\left(\mu+P_{r}\right) A \sigma,
$$

whose general solution is

$$
X_{T F}=-\frac{4 \pi \int_{0}^{t}\left[2 \Pi \dot{R}-\left(\mu+P_{r}\right) A R \sigma\right] R^{2} d t}{R^{3}} .
$$

Thus our system is initially homogeneous in the energydensity, however as time goes on this condition will be affected by the anisotropy of the pressure and the shear of the fluid according to (59).

\section{Dissipative geodesic dust.}

Finally, in order to bring out the effect of dissipation in the formation of energy-density inhomogeneities we shall consider the case of dissipative geodesic dust. Observe that in the dissipative case the dust condition does not imply that the fluid is geodesic as can be easily seen from (35), therefore the geodesic condition here is not redundant and is assumed, both, for sake of simplicity and also in order to isolate the effects produced by dissipative phenomena alone. It is worth mentioning that a great deal of work has been done in order to find exact solutions describing radiating geodesic dust configurations (e.g. see [46]-53] and references therein)

Thus we have $P_{r}=P_{\perp}=0$ and $A=1$, in which case our equations (36) and (37) become

$$
(\mathcal{E}-4 \pi \tilde{\mu})+\frac{3 \dot{R}}{R}(\mathcal{E}-4 \pi \tilde{\mu})-\frac{12 \pi \tilde{q} R^{\prime}}{B R}=0,
$$

and

$$
(\mathcal{E}-4 \pi \tilde{\mu})^{\prime}=-\frac{3 R^{\prime}}{R} \mathcal{E}-\frac{12 \pi \tilde{q} \dot{R} B}{R} .
$$

From the equation above it follows that the quantity that now determines the existence of energy-density inhomogeneities is:

$$
\Psi \equiv \mathcal{E}+\frac{12 \pi \int_{0}^{r} \tilde{q} B \dot{R} R^{2} d r}{R^{3}} .
$$

Indeed, from (61) is not difficult to find that $\tilde{\mu}^{\prime}=0 \leftrightarrow$ $\Psi=0$.

Then, an equation for the evolution of $\Psi$ can be obtained from (60), using (12) and (34):

$$
\dot{\Psi}-\frac{\dot{\Omega}}{R^{3}}=-4 \pi \tilde{\mu} \sigma-\frac{4 \pi \tilde{q}^{\prime}}{B}+\frac{4 \pi \tilde{q} R^{\prime}}{B R}-\frac{3 \Psi \dot{R}}{R},
$$

with $\Omega \equiv 12 \pi \int_{0}^{r} \tilde{q} B \dot{R} R^{2} d r$.

The general solution of (63) is

$$
\Psi=\frac{\int_{0}^{t}\left(\dot{\Omega}-4 \pi \tilde{\mu} \sigma R^{3}-\frac{4 \pi R^{3} \tilde{q}^{\prime}}{B}+\frac{4 \pi R^{2} \tilde{q} R^{\prime}}{B}\right) d t}{R^{3}},
$$

where the influence of diferent factors on the evolution of $\Psi$ are clearly indicated.

We can further transform the expression above by noticing that in the case under consideration (35) can be formally integrated to obtain

$$
\tilde{q}=\frac{\phi(r)}{B^{2} R^{2}},
$$

where $\phi(r)$ is an arbitrary function satisfying $\phi(0)=0$.

Thus we see from (64) that starting from an homogeneous configuration the appearance of density inhomogeneities will depend on two different factors; on the one hand the shear of the fluid and on the other dissipative 
terms. In order to isolate further the influence of these latter terms, let us consider the shear-free case.

If the shear is assumed to vanish, then it is a simple matter to see that we may put $R=B r$, then using (65), we obtain from (64)

$$
\Psi=\frac{\int_{0}^{t}\left[4 \pi \int_{0}^{r} \dot{\Theta} \phi(r) r d r-\frac{4 \pi \phi(r)^{\prime} r^{3}}{R^{2}}+\frac{8 \pi \phi(r) r^{2}}{R^{3}}\left(\frac{5 R^{\prime} r}{2}-R\right)\right] d t}{R^{3}}
$$

from the above it is apparent that dissipation affect $\Psi$ through, both, local and non-local tems.

Finally it is instructive (always in the shear-free case) to bring out the role of relaxational effects in the evolution of $\Psi$. Thus we shall assume that we are in the purely diffusion approximation $(\epsilon=0)$ implying $\tilde{q}=q ; \tilde{\mu}=\mu$ Thus, from (35) it follows

$$
\dot{q}=-\frac{4 q \Theta}{3},
$$

then combining the above equation with (40) we obtain

$$
q=-\kappa \frac{r T^{\prime}}{R\left(1-\frac{4 \Theta \tau}{3}\right)},
$$

or using (65)

$$
\phi(r)=-\kappa \frac{R^{3} T^{\prime}}{r\left(1-\frac{4 \Theta \tau}{3}\right)} .
$$

Feeding back (69) into (66) we can evaluate the influence of $\tau$ on the evolution of $\Psi$ for any specific configuration.

\section{CONCLUSIONS}

We have identified the different aspects of the fluid distribution which are responsible for the appearance of energy-density inhomogeneities and have found the evolution equation for the variables representing those aspects.
For dust and isotropic perfect fluids the relevant factor is the Weyl tensor, therefore the stability of the homogeneous energy-density condition is equivalent to the stability of the conformal flatness condition.

However for locally anisotropic fluids, inhomogeneities are also related to the local anisotropy of pressure. The specific variable related to the existence of energydensity inhomogeneities was found as well as its evolution equation. That specific variable was identified as one of the structure scalars.

Dissipation emerges also as a source of energy-density inhomogeneities. In order to isolate the effect of dissipation from others factors we have considered the dust, geodesic case, and as a particular subcase we studied further the shear-free case. The non-local contributions of dissipation to the formation of inhomogeneities and the role of relaxational effects are the most relevant results of this part of the study.

To conclude, the following remark is in order: The fact that the different variables considered here affect the formation of energy-density inhomogeneities might be intuitively clear, however the specific form in which these variables do so, is far from obvious. Indeed, we may consider a situation in which any of the above mentioned variables are present but are such that their combinations in (44), (59) or (66) vanish. In this latter case the homogeneous energy-density condition will be stable in spite of the fact that all (or some ) of the mentioned factors are present.
[1] D. M. Eardley and L. Smarr, Phys.Rev. D 19, 2239 (1979).

[2] D. Christodoulou Commun. Math. Phys. 93, 171 (1984).

[3] R. P. A. C. Newman Class. Quantum Grav. 3, 527 (1986).

[4] B. Waugh and K. Lake, Phys.Rev. D 38, 1315 (1988).

[5] L. Herrera, A. Di Prisco, J. Hernandez-Pastora and N. O. Santos Phys. Lett. A 237, 113 (1998).

[6] L. Herrera and A. Di Prisco Gen. Rel. Grav., 31, 301 (1999).

[7] F. Mena and R. Tavakol, Class. Quantum Grav. 16, 435 (1999).
[8] G. Pinheiro and R. Chan Gen. Rel. Grav. 43, 1451 (2011).

[9] R. Penrose, General Relativity, An Einstein Centenary Survey, Ed. S. W. Hawking and W. Israel (Cambridge: Cambridge University Press) p. 581-638 (1979).

[10] L. Herrera, A. Di Prisco, J. Martin, J. Ospino, N. O. Santos and O. Troconis Phys. Rev. D 69, 084026 (2004).

[11] A Di Prisco, L. Herrera, G. Le Denmat, M. MacCallum and N.O. Santos, Phys. Rev. D 76, 064017, (2007).

[12] M. Sharif and A. Siddiqa arXiv:1008.0695

[13] N. Pelavas and K. Lake arXiv:gr-qc/9811085 
[14] W. B. Bonnor Phys. Lett. A 122, 305 (1985).

[15] S. W. Goode, A. A. Coley and J. Wainwright Class. Quantum Grav. 9, 445 (1992).

[16] G. F. R. Ellis, Relativistic Cosmology in: Proceedings of the International School of Physics "Enrico Fermi", Course 47: General Relativity and Cosmology. Ed. R. K. Sachs (Academic Press, New York and London) (1971).

[17] G. F. R. Ellis, Gen. Rel. Grav. 41, 581 (2009).

[18] R. Maartens Phys. Rev. D 55, 463 (1997).

[19] G. F. R. Ellis and H. van Elst arXiv: $g r-q c / 9812046$.

[20] R. Sussman arXiv:0801.3324v4.

[21] L. Herrera, J. Ospino, A. Di Prisco, E. Fuenmayor and O. Troconis, Phys. Rev. D 79, 064025 (2009).

[22] L. Herrera, A. Di Prisco and J. Ospino Gen.Rel. Grav.42, 1585 (2010).

[23] L. Herrera and N. O. Santos Phys. Rev. D 70084004 (2004).

[24] B. Ivanov, Int. J. Theor. Phys. 49, 1236 (2010).

[25] L. Herrera and N. O. Santos Phys. Rep. 286, 53 (1997).

[26] G. Darmois Mémorial des Sciences Mathématiques (Gauthier-Villars, Paris, 1927) Fasc. 25.

[27] C. Misner and D. Sharp, Phys. Rev. 136, B571 (1964).

[28] M. Cahill and G. McVittie, J. Math. Phys. 11, 1382 (1970).

[29] L. Herrera, N. O. Santos and A. Wang Phys. Rev. D 78, 084026 (2008).

[30] I Müller Z. Physik 198, 329 (1967).

[31] W Israel Ann. Phys. (NY) 100, 310 (1976).

[32] W. Israel and J. Stewart Phys. Lett. A 58. 213 (1976).

[33] W. Israel and J. Stewart Ann. Phys. (NY) 118, 341 (1979).

[34] D Joseph and L Preziosi Rev. Mod. Phys. 61, 41 (1989).

[35] D. Jou, J Casas-Vázquez and G Lebon Rep. Prog. Phys. 51, 1105 (1988).
[36] R Maartens astro-ph/9609119

[37] L Herrera and D Pavón Physica A, 307, 121 (2002).

[38] W Hiscock and L Lindblom Ann. Phys. (NY) 151, 466 (1983).

[39] C Eckart Phys. Rev. 58, 919 (1940).

[40] L Landau and E Lifshitz, Fluid Mechanics (Pergamon Press, London) (1959).

[41] D Pavón, D Jou and J Casas-Vázquez Ann. Inst. H Poincaré A36, 79 (1982).

[42] B Carter Journées Relativistes, ed. M Cahen, R Debever and J Geheniau, (Université Libre de Bruxelles) (1976).

[43] C Cattaneo Atti Semin. Mat. Fis. Univ. Modena 3, 3 (1948).

[44] L. Herrera and N. O. Santos Mon. Not. R. Astr. Soc. 287, 161 (1997).

[45] J. Triginer and D. Pavon Class. Quantum Grav. 12, 689, (1995).

[46] L. Herrera, A. Di Prisco, J. Ospino and J. Carot Phys. Rev. D 82, 024021 (2010).

[47] C. Kolassis, N. O. Santos and D. Tsoubelis Astrophys. J. 327, 755 (1988).

[48] M. Govender, S. Maharaj and R. Maartens Class. Quantum Grav. 15, 323 (1998).

[49] S. Maharaj, M. Govender Int. J. Mod. Phys. D 14, 667 (2005).

[50] S. Thirukkanesh and S. Maharaj J. Math. Phys. 50, 022502 (2009).

[51] N. Naidu, M. Govender and K. Govinder Int. J. Mod. Phys. D 15, 1053 (2006).

[52] S. Rajah and S. Maharaj J. Math. Phys. 49, 012501 (2008).

[53] S. Thirukkanesh and S. Maharaj J. Math. Phys. 51, 072502 (2010). 